\title{
Toll-like receptor 4 and high-mobility group box-1 are involved in ictogenesis and can be targeted to reduce seizures
}

\author{
Mattia Maroso $^{1}$, Silvia Balosso ${ }^{1}$, Teresa Ravizza ${ }^{1}$, Jaron Liu ${ }^{2}$, Eleonora Aronica ${ }^{3,4}$, Anand M Iyer ${ }^{3}$, Carlo Rossetti ${ }^{5,6}$, \\ Monica Molteni ${ }^{6}$, Maura Casalgrandi ${ }^{7}$, Angelo A Manfredi ${ }^{8}$, Marco E Bianchi ${ }^{2}$ \& Annamaria Vezzani ${ }^{1}$
}

\begin{abstract}
Brain inflammation is a major factor in epilepsy, but the impact of specific inflammatory mediators on neuronal excitability is incompletely understood. Using models of acute and chronic seizures in C57BL/6 mice, we discovered a proconvulsant pathway involving high-mobility group box-1 (HMGB1) release from neurons and glia and its interaction with Toll-like receptor 4 (TLR4), a key receptor of innate immunity. Antagonists of HMGB1 and TLR4 retard seizure precipitation and decrease acute and chronic seizure recurrence. TLR4-defective $\mathrm{C} 3 \mathrm{H} / \mathrm{HeJ}$ mice are resistant to kainate-induced seizures. The proconvulsant effects of HMGB1, like those of interleukin-1 $\beta$ (IL-1 $\beta$ ), are partly mediated by ifenprodil-sensitive $N$-methyl-D-aspartate (NMDA) receptors. Increased expression of HMGB1 and TLR4 in human epileptogenic tissue, like that observed in the mouse model of chronic seizures, suggests a role for the HMGB1-TLR4 axis in human epilepsy. Thus, HMGB1-TLR4 signaling may contribute to generating and perpetuating seizures in humans and might be targeted to attain anticonvulsant effects in epilepsies that are currently resistant to drugs.
\end{abstract}

Epilepsy is a disabling neurological disorder characterized by recurring, unprovoked seizures. It affects about $1 \%$ of the population of all ages and often requires lifelong medication ${ }^{1}$. In about $30 \%$ of affected individuals, epilepsy is refractory to pharmacological treatment ${ }^{2}$, and surgical removal of the epileptic focus is suitable only for a minority of them. Understanding the molecular events underlying the occurrence of seizures is necessary for devising new therapeutic approaches.

Increasing evidence supports the involvement of inflammatory and immune processes in the etiopathogenesis of seizures ${ }^{3}$. Inflammatory responses induced by brain-damaging events such as neurotrauma, stroke, infection, febrile seizures and status epilepticus are associated with acute symptomatic seizures and a high risk of developing epilepsy ${ }^{4,5}$. Pronounced inflammatory processes have been described in epileptogenic brain tissue from drug-resistant patients with temporal lobe epilepsy (TLE) ${ }^{6-8}$ and epilepsies associated with developmental malformations of the cortex ${ }^{9,10}$. Pharmacological and genetic studies in animal models have shown that specific inflammatory mediators such as cytokines, complement factors and prostaglandins substantially contribute to seizures and that interfering with these molecules or their receptors can reduce seizure frequency and severity ${ }^{11}$.

We previously showed in rats and mice that the proinflammatory cytokine IL-1 $\beta$ is rapidly upregulated during seizures in microglia, astrocytes and endothelial cells in the epileptic focus as well as in forebrain regions recruited in epileptic activity ${ }^{8,12-14}$. IL-1 $\beta$ exerts powerful proconvulsant actions via a signaling pathway in neurons involving its receptor IL-1R1, the IL-1R accessory protein and myeloid differentiation primary response protein (MyD88) complex and Src family kinases, leading to NMDA receptor-2B (NR2B) phosphorylation and enhanced NMDA-dependent $\mathrm{Ca}^{2+}$ influx ${ }^{12,15,16}$.

The pathway activated by IL- $1 \beta$ depends on MyD88, but other surface receptors can recruit MyD88, notably TLRs, which have a key role in pathogen recognition ${ }^{17}$. TLRs recognize various molecules of microbial origin, called pathogen-associated molecular patterns (PAMPs), and trigger inflammation by inducing the transcription of genes encoding cytokines, including IL-1 $\beta$. TLR4 in particular detects lipopolysaccharide (LPS), a major outer membrane component of Gram-negative bacteria. Given that TLR4 is expressed in the brain $^{18}$ and LPS lowers the seizure threshold in rodents ${ }^{19,20}$, we sought to investigate whether TLR4 has a role in the onset and recurrence of seizures.

Increasing evidence indicates that, in the absence of pathogens, TLR signaling can be activated by molecules released by injured tissue $^{21}$. These molecules, named damage-associated molecular patterns (DAMPs), include HMGB1, a nearly ubiquitous chromatin component that is passively released by necrotic cells, retained by

\footnotetext{
${ }^{1}$ Department of Neuroscience, Mario Negri Institute for Pharmacological Research, Milano, Italy. ${ }^{2}$ Department of Genetics and Cell Biology, San Raffaele University and San Raffaele Research Institute, Milano, Italy. ${ }^{3}$ Department (Neuro) Pathology, Academisch Medisch Centrum, Amsterdam, The Netherlands. ${ }^{4}$ The Netherlands Foundation (Stichting Epilepsie Instellingen Nederland), Heemstede, The Netherlands. ${ }^{5}$ Department of Biotechnology and Molecular Sciences, University of Insubria, Varese, Italy. ${ }^{6}$ Department of Environmental Health Sciences, Mario Negri Institute for Pharmacological Research, Milano, Italy. ${ }^{7} \mathrm{HMGBiotech}$ srl, Milano, Italy. ${ }^{8}$ Department of Regenerative Medicine, San Raffaele University and San Raffaele Research Institute, Milano, Italy. Correspondence should be addressed to A.V. (vezzani@marionegri.it).
} 
Figure 1 HMGB1 and TLR4 immunoreactivity in the CA1 pyramidal layer of hippocampi of kainic acid-injected C57BL/6 mice. (a-c) Photomicrographs of hippocampi injected with vehicle (a), or $1 \mathrm{~h}(\mathbf{b})$ and $3 \mathrm{~h}$ (c) after kainic acid-induced seizures. Top two rows, HMGB1 immunoreactivity in nuclei of pyramidal neurons and cells (arrows) of the strata radiatum (Rad) and lacunosum-molecolare ( $\mathrm{LMol}$ ); some cells with neuronal morphology (green arrows) show cytoplasmic immunoreactivity. Cells with astrocytic morphology $(\mathbf{b}, \mathbf{c})$ show HMGB 1 in the cytoplasm (arrowheads). Bottom row, HMGB1 signal only (left) and colocalization of HMGB 1, DNA (Hoechst), GFAP for astrocytes (middle) and CD11b for microglia-like cells (right). GFAP-positive cells show HMGB1 in nuclei (a) and around nuclei (b,c).

(d) Quantification of HMGB1-positive cells in control-injected hippocampi (C), $1 \mathrm{~h}$ and $3 \mathrm{~h}$ after seizures (means \pm s.e.m., $n=4$ ). Nuclear staining: ${ }^{*} P<0.05$ versus control; $\# P<0.05$ versus $1 \mathrm{~h}$; Extranuclear staining: $\$ P<0.05$ versus $1 \mathrm{~h}$ and control; one-way analysis of variance (ANOVA) followed by Tukey's test. ND, not detectable.

(e) Quantification of western blots for HMGB1 in mouse hippocampal homogenate from control mice (C) and mice $3 \mathrm{~h}$ after kainic acid seizures. Error bars (means \pm s.e.m., $n=5$ ) represent the ratios of the optical densities of the HMGB1 and glyceraldehyde3-phosphate dehydrogenase (GAPDH) bands; ${ }^{*} P<0.05$ by Mann-Whitney test. ( $\left.\mathbf{f}-\mathbf{i}\right)$ TLR4 immunoreactivity in hippocampi injected with vehicle (f) or $1 \mathrm{~h} \mathrm{(g)} \mathrm{or} 3 \mathrm{~h}(\mathbf{h})$ after kainateinduced seizures. Arrows in $\mathbf{g}$ and $\mathbf{h}$ point to neurons. Colocalization of TLR4 with cell-specific markers ( $g, h$, second row) in NeuN-positive neurons, GFAP-positive astrocytes, CD11b-positive microglia-like cells. (i) TLR4

immunoreactivity in CD11b-positive cells in mouse hippocampus after intracerebroventricular lipopolysaccharide injection. MoDG, molecular layer of dentate gyrus; 3V, third ventricle. Scale bars: a-c, f-h (top row) $75 \mu \mathrm{m}$; a-c (middle row) $25 \mu \mathrm{m}$; a-c (bottom row), g,h (bottom row) and i, $15 \mu \mathrm{m}$. a
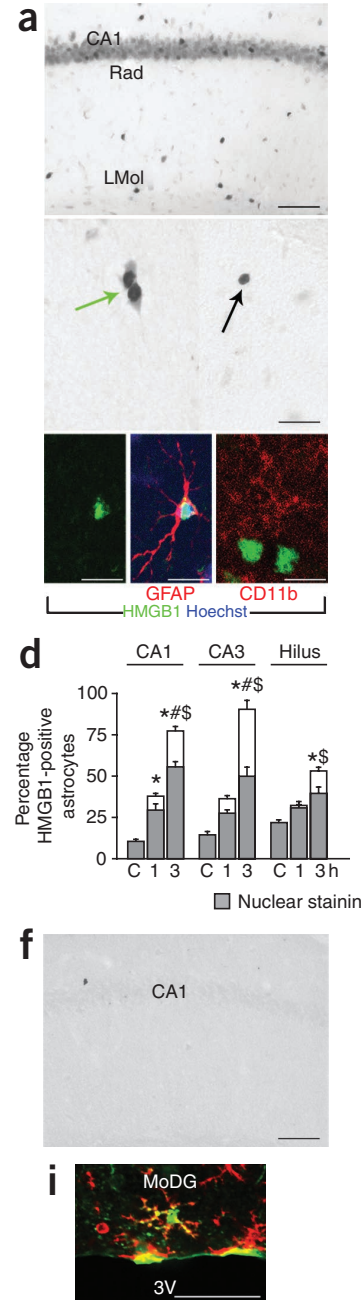

TLR4 CD11b b
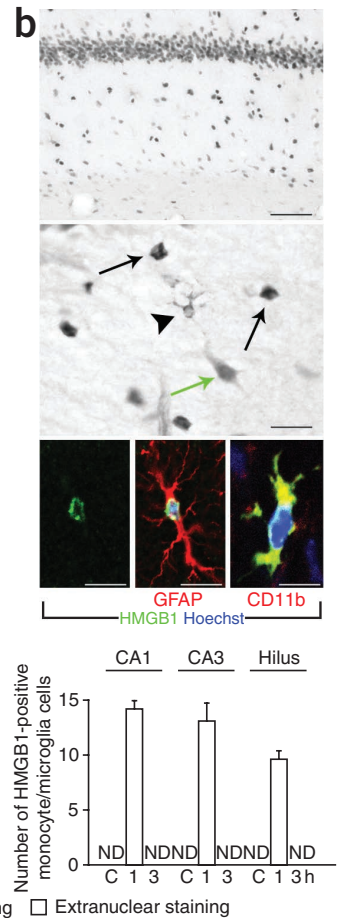

g

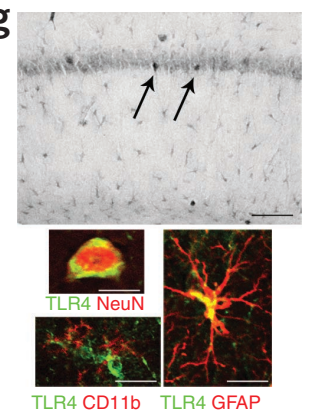

C

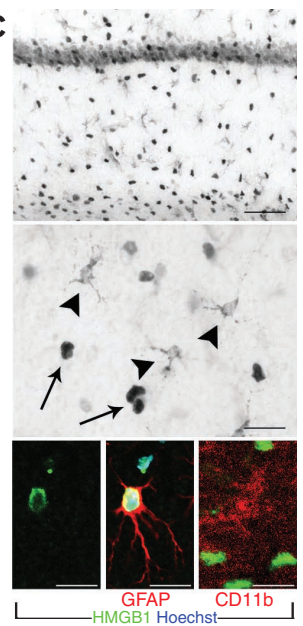

e

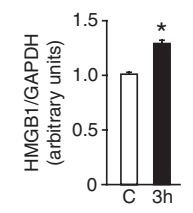

h

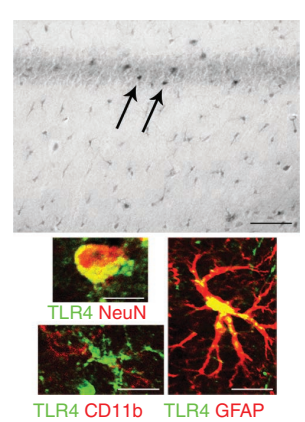

cells undergoing programmed death (apoptosis) and actively secreted by cells in profound distress ${ }^{22,23}$. HMGB1 secretion does not follow the canonical endoplasmic reticulum-Golgi pathway and occurs via relocation of the nuclear protein to the cytoplasm ${ }^{24}$. Extracellular HMGB1 binds the receptor for advanced glycation end products ${ }^{22}$ and several other receptors, including TLR4 ${ }^{25,26}$.

We asked whether proconvulsive stimuli could lead to HMGB1 release and TLR4 activation, thereby contributing to seizures. Our results indicate that HMGB1 and TLR4 contribute to the generation and severity of seizures, which therefore can be reduced by TLR 4 and HMGB1 antagonists. Studies in spontaneously epileptic mice and in human TLE tissue indicate that the HMGB1-TLR4 axis also contributes to seizures in chronic epilepsy.

\section{RESULTS}

\section{Expression of HMGB1 and TLR4 in mouse models of seizures}

We first investigated whether seizure activity affects the expression of HMGB1 and TLR4. We used two mouse models of focal-onset acute seizures induced by unilateral intrahippocampal injection of kainic acid (7 ng) or bicuculline (51 ng) (Supplementary Fig. 1). Kainic acid is an agonist of one class of glutamate receptors, whereas bicuculline is an antagonist of $\gamma$-aminobutyric acid A receptors; they trigger seizures by increasing excitatory neurotransmission and decreasing inhibitory neurotransmission, respectively. In these models, the seizure activity is similar and occurs to the same extent in the injected and contralateral side of the hippocampus. Kainic acid at low dose causes excitotoxic cell damage only to pyramidal cells in the CA3 area of the injected hippocampus $^{16,27}$, whereas bicuculline provokes seizures in the absence of neurodegeneration ${ }^{28}$. We also used an established mouse model of chronic epilepsy, where seizure activity develops within 1 week after intrahippocampal application of $200 \mathrm{ng}$ kainic acid ${ }^{29,30}$. This model recapitulates the major neuropathological features of human TLE (Supplementary Fig. 2) and, in particular, recurring spontaneous seizures that do not respond to various anticonvulsant drugs ${ }^{29}$.

We investigated the distribution of the immunohistochemical signal of HMGB1 in mice injected with kainic acid (Fig. 1a-d). In control hippocampi, HMGB1 is present mostly in nuclei of the pyramidal neurons (Fig. 1a) and granule cells of the dentate gyrus (data not shown). In the strata radiatum, lacunosum-moleculare and moleculare, we observed scattered cells with nuclear staining as well as neurons with both nuclear and cytoplasmic staining; most cells were HMGB1 negative (Fig. 1a). Between 1 and $3 \mathrm{~h}$ after the onset of 
a

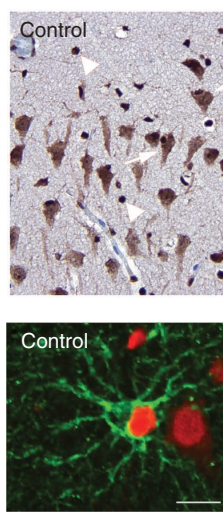

HMGB1 GFAP
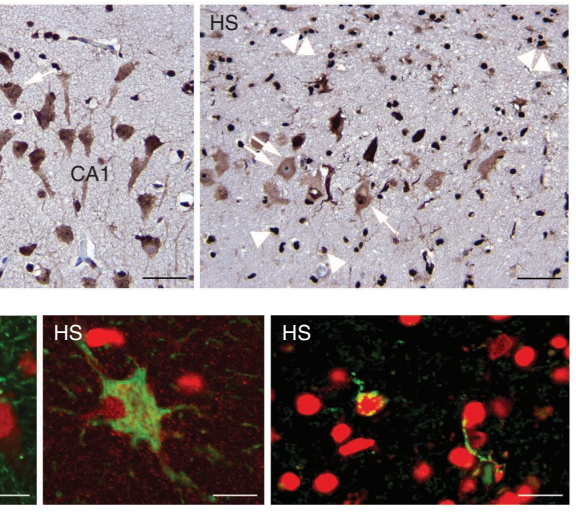

HMGB1 GFAP

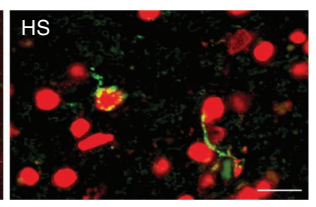

HMGB1 HLA-DR

Figure 2 HMGB1 and TLR4 immunoreactivity in the hippocampi of control subjects and subjects with TLE-HS. (a) Immunohistochemical staining for HMGB1 in the CA1 region of control individuals and individuals with TLE. Arrows indicate pyramidal neurons. Double arrows point to neurons with prominent cytoplasmic immunoreactivity; arrowheads point to cells with glial morphology with nuclear staining and double arrowheads point to cells with glial morphology with cytoplasmic staining. (b) Immunofluorescence of HMGB1, GFAP and HLA-DR in hippocampi from control individuals and individuals with TLE-HS.

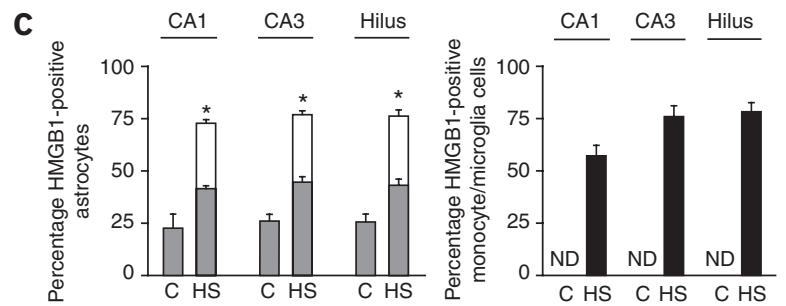

$\square$ Nuclear staining $\square$ Extranuclear staining $\square$ Nuclear + extranuclear staining

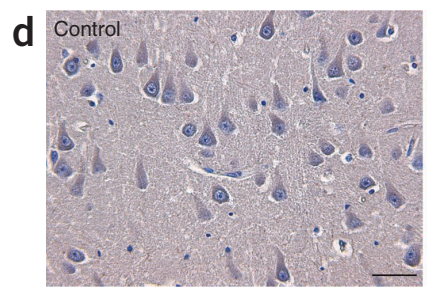

e
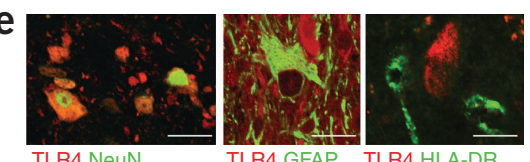

TLR4 GFAP TLR4 HLA-DR
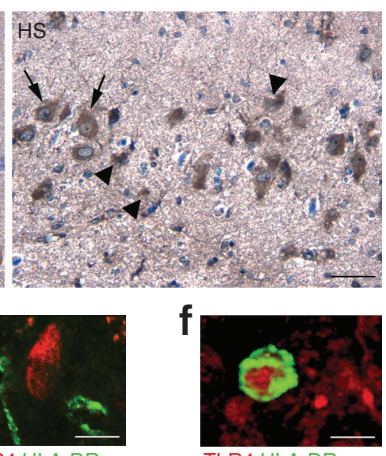

TLR4 HLA-DR

(c) Quantification of HMGB1-positive cells in control subjects $(C)$ and subjects with TLE-HS (means \pm s.e.m., $n=6$ ). Extranuclear staining: ${ }^{*} P<0.05$ versus control, one-way ANOVA followed by Tukey's test. (d) TLR4 immunostaining in the CA1 region; arrows point to neurons and arrowheads to reactive glial cells. (e) Confocal images showing colocalization of TLR4 with NeuN in neuronal cells, or with GFAP in reactive astrocytes, and lack of colocalization of TLR4 with HLA-DR in cells of the microglia/macrophage lineage. (f) TLR4 staining in HLA-DR-positive cells in a brain tissue sample of a subject with multiple sclerosis. Sections in $\mathbf{a}$ and $\mathbf{d}$ are counterstained with hematoxylin. Scale bars: $\mathbf{a}, \mathbf{d}, 50 \mu \mathrm{m} ; \mathbf{b}, \mathbf{e}, \mathbf{f}, 20 \mu \mathrm{m}$.

seizures, we found a progressive increase in nuclear and perinuclear HMGB1 staining in astrocytes (Fig. 1a-c) in all subfields of injected (Fig. 1d) and contralateral hippocampi (Supplementary Fig. 3b). Although we did not detect HMGB1 in cells with microglial morphology in control hippocampi, a short-lived wave of cytoplasmic expression in microglia appeared $1 \mathrm{~h}$ after seizure onset and was again absent after $3 \mathrm{~h}$ (Fig. 1a-d). In agreement with immunohistochemical results, we measured, by western blotting, an average $27 \%$ increase in HMGB1 abundance in kainic acid-injected hippocampi $3 \mathrm{~h}$ after the onset of seizures (Fig. 1e). This increase in the whole tissue represents a substantial increase in the fraction of cells affected.

The induction of HMGB1 in the hippocampus after bicucullineinduced seizures was bilateral and similar to that described for kainic acid-induced seizures (Supplementary Figs. 3 and 4), although the changes were less prominent. In chronic epileptic mice, the cell pattern and extent of HMGB1 expression in hippocampi (Supplementary Figs. 3 and $\mathbf{5}$ ) were similar to those described after acute kainic acidinduced seizures.

We did not find a parallel change in the number of neurons showing nuclear staining, cytoplasmic staining or both in any seizure model (data not shown). All changes observed in the CA1 region were similar in the CA3 region and in dentate gyrus (data not shown).

We next investigated TLR4 expression (Fig. 1f-h and Supplementary Figs. 4 and 5). Whereas we found no signal in control slices (Fig. 1f), we observed substantial TLR 4 expression $1 \mathrm{~h}$ (Fig. 1g) and $3 \mathrm{~h}$ (Fig. 1h) after seizure onset in neurons within the pyramidal layers; glial fibrillary acidic protein (GFAP)-positive astrocytes also expressed TLR4 in the various hippocampal subfields including CA1 (Fig. 1g,h), CA3 and hilus (data not shown). TLR4 was not expressed in CD11b-positive cells of microglial morphology (Fig. 1g,h). As a positive control, we did detect TLR4 in CD11b-positive cells in the hippocampus after intraventricular LPS injection (Fig. 1i). We observed a similar pattern of
TLR4 induction in both neurons and astrocytes, but not in microglia, in chronic epileptic mice (Supplementary Fig. 5). In bicuculline-injected mice, TLR4 staining increased bilaterally in neurons, but not in astrocytes, $1.5 \mathrm{~h}$ after the onset of seizures, when epileptic activity had just abated (Supplementary Fig. 4). These differences from the kainic acid model may be due to the lack of cell loss, the shorter duration of seizures (90 min bicuculline versus 120 min kainic acid) or both.

\section{Expression of HMGB1 and TLR4 in human epileptogenic tissue} Having characterized a specific pattern of HMGB1 and TRL4 expression in the hippocampi of chronic epileptic mice, we looked for a similar pattern in hippocampal specimens obtained at surgery in subjects with drug-resistant TLE with hippocampal sclerosis (TLE-HS) (Fig. 2). In autoptic control tissue, we detected HMGB1 signal in nuclei of cells identified a posteriori as neurons and astrocytes, as well as in neuronal cytoplasm (Fig. 2a). In TLE-HS, cytoplasmic HMGB1 staining was substantially increased in GFAP-positive astrocytes as well as in human leukocyte antigen DR (HLA-DR)-positive processes (Fig. 2a-c).

TLR4 was undetectable in autoptic control hippocampi, whereas it was clearly detectable in pyramidal neurons and GFAP-positive astrocytes in TLE-HS specimens (Fig. 2d). HLA-DR-positive microglia-like cells did not show TLR4 staining (Fig. 2e), whereas HLADR-positive cells in multiple sclerosis specimens did stain for TLR4 (Fig. 2f). We found similar patterns in the CA3 and hilus of the dentate gyrus (data not shown). HMGB1 and TLR4 staining in surgical hippocampal specimens from subjects with focal epileptogenic lesions not involving the hippocampus proper was similar to that in autoptic control tissue (data not shown).

\section{HMGB1 promotes seizures in a TLR4-dependent way}

HMGB1 and TLR4 form a ligand-receptor pair. To examine whether TLR4 activation by HMGB1 contributes to ictogenesis, we used 

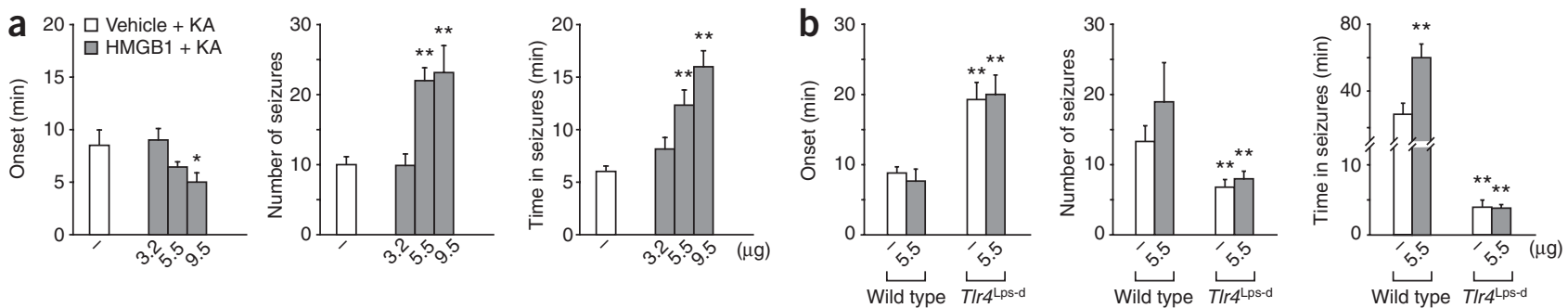

Figure 3 Dose-dependent proconvulsant effect of HMGB1 in wild-type mice and absence of HMGB1 effect in TIr4Lps-d mice. (a) Seizure parameters (onset, number and duration) in C57BL/6 mice ( $n=8$ or 9) injected in the hippocampus with HMGB1 at the indicated doses, 15 min before kainic acid (KA) injection. Vehicle + KA group represents mice receiving the corresponding volume of PBS before kainic acid. Error bars are means \pm s.e.m.; ${ }^{*} P<0.05$, ** $P<0.01$ versus vehicle + KA by one-way ANOVA followed by Tukey's test. (b) Seizure parameters (onset, number and duration) in mice defective in TLR4 signaling (TIr4Lps-d) and the corresponding wild-type mice ( $n=6$ each group) after injection in the hippocampus with $5.5 \mu \mathrm{g}$ HMGB1 or the corresponding volume of vehicle followed 15 min later by kainic acid injection. Error bars are means \pm s.e.m., $* * P<0.01$ versus wild type by two-way ANOVA followed by Tukey's test.

pharmacological and genetic approaches. First, we increased extracellular HMGB1 amounts in C57BL/6 mice by intrahippocampal injection of recombinant HMGB1 15 min before kainic acid application (Fig. 3a). HMGB1 addition (5.5 and $9.5 \mu \mathrm{g}$ per mouse) increased the frequency of kainic acid-induced seizures by about 2.5-fold (representative electroencephalogram (EEG) tracings in Supplementary Fig. 1a-d) and the total time spent in seizures. The time of onset of the first seizure was markedly reduced by $9.5 \mu \mathrm{g}$ HMGB1. An HMGB1 dose of $3.2 \mu \mathrm{g}$ per mouse was ineffective.

We next investigated whether HMGB1 signals through TLR4 by using $\mathrm{C} 3 \mathrm{H} / \mathrm{HeJ}$ mice, which have a spontaneous mutation in the Toll/IL-1 receptor domain of TLR4 (Tlr4 $\left.4^{\mathrm{Lps}-\mathrm{d}}\right)$, leading to functional inactivation of the receptor ${ }^{31}$. HMGB1 (5.5 $\mu \mathrm{g}$ per mouse) had no effect on kainic acid-induced seizures when injected into $\mathrm{C} 3 \mathrm{H} / \mathrm{HeJ}$ mice but was proconvulsant in their wild-type $\mathrm{C} 3 \mathrm{H} / \mathrm{HeouJ}$ counterparts, increasing the time spent in seizures by about twofold (Fig. $3 \mathbf{b}$ and Supplementary Fig. 1f-i). C3H/HeJ mice also had a reduced intrinsic susceptibility to seizures, as shown by a delay in kainic acid-induced seizure onset and a substantial reduction in seizure frequency and total duration (Fig. 3b).

\section{Overstimulated neurons release HMGB1}

A high local concentration of glutamate, resulting from hyperexcitation of the neuronal network, is thought to have a key role in the initiation and spread of seizures. We thus hypothesized that overstimulation of glutamate receptors might induce neurons to release HMGB1.

We exposed mixed primary cocultures of neurons and glia to $0.25 \mathrm{mM}$ glutamate for $2 \mathrm{~h}$ then fixed and immunostained the cells
(Supplementary Fig. 6). HMGB1 was located only in the nuclei of unchallenged neurons and glial cells; after glutamate exposure, HMGB1 signal was still present only in the nuclei of glial cells, but in neurons it was located in both the nucleus and the cytoplasm (neuNpositive cells, Supplementary Fig. 6a). We confirmed these results in primary cortical neurons infected with a lentivirus expressing an HMGB1-GFP fusion protein. Unchallenged neurons contained HMGB1-GFP only in the nucleus, whereas neurons exposed to $0.25 \mathrm{mM}$ glutamate had a large amount of HMGB1-GFP throughout the cell bodies and processes (Supplementary Fig. 6b).

Neurons overstimulated by glutamate eventually undergo excitotoxic death. Cortical neurons exposed in vitro to $0.25 \mathrm{mM}$ glutamic acid started to die after about $2 \mathrm{~h}$ and were almost completely dead after 24 h, as judged morphologically (Supplementary Fig. 6c) and by the release of lactate dehydrogenase (Supplementary Fig. 6d). Notably, HMGB1 was also released into the medium (Supplementary Fig. 6d). We showed previously that HMGB1 retention upon apoptosis depends on caspase- 3 activation ${ }^{32}$; caspase- 3 was not activated by glutamate exposure, nor was DNA fragmented (ref. 33 and data not shown).

HMGB1 and TLR4 antagonists reduce acute and chronic seizures Overall, our findings predict that pharmacological blockade of the HMGB1-TLR4 axis should reduce the frequency and duration of seizures. We then interfered with the activity of endogenous HMGB1 by injecting BoxA, a fragment of HMGB1 with antagonistic activity ${ }^{34-36}$. BoxA injection ( $7.5 \mu$ g per mouse) delayed the onset of seizures (Fig. 4a), and 2.5 and $7.5 \mu \mathrm{g}$ of BoxA significantly $\square$ Vehicle + KA $\square$ Lps-Rs + KA

$\square$ BoxA + KA $\square$ Cyp + KA

a

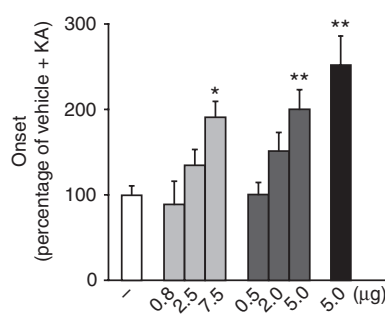

b

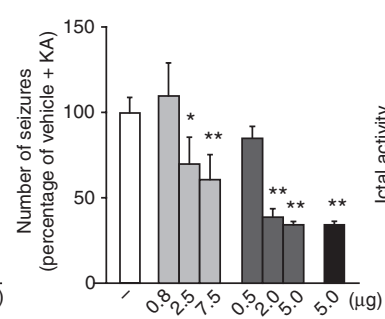

C

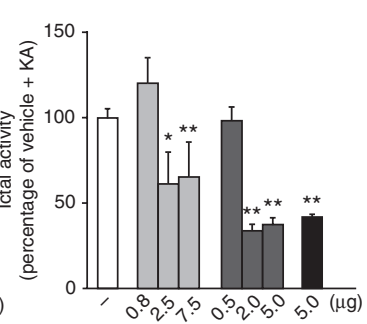

$\square$ Vehicle + bicuculline BoxA + bicuculline $\square$ Lps-Rs + bicuculline

d

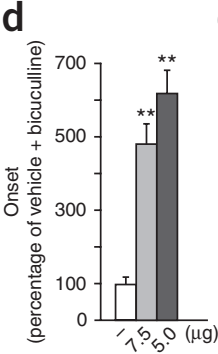

e

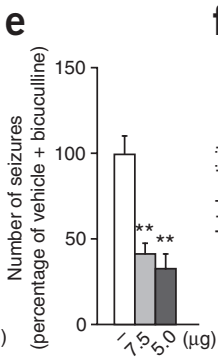

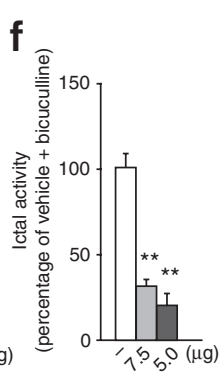

Figure 4 Anticonvulsant effects of BoxA, Lps-Rs and Cyp in acute seizure models. (a-f) Seizure parameters (onset, a,d; number, b,e; duration, c,f) in mice injected in the hippocampus with vehicle or the various drugs at the doses indicated, followed 15 min later by kainic acid (a-c) or bicuculline injection ( $\mathbf{d}-\mathbf{f}$ ). Error bars (means \pm s.e.m., $n=8-11$ each group) represent percentage changes in inhibitor-treated mice versus mice injected with vehicle + KA (onset $8.5 \pm 1.4 \mathrm{~min}$, number of seizures $10.0 \pm 1.0$, ictal activity $6.3 \pm 0.5 \mathrm{~min}$ ) or vehicle + bicuculline (onset $1.9 \pm 0.3 \mathrm{~min}$, number of seizures $13.0 \pm 1.0$, ictal activity $9.1 \pm 0.7 \mathrm{~min}) .{ }^{*} P<0.05,{ }^{* *} P<0.01$ versus vehicle by one-way ANOVA followed by Tukey's test. 
Figure 5 Anticonvulsant effects of BoxA and Lps-Rs in the chronic seizure model. (a) Experimental protocol. EEG seizures were recorded in eight C57BL/6 mice with a stable baseline of spontaneous seizures (Online Methods and Supplementary Fig. 2 contain details). The first EEG recording period $(0-2 h)$ was used to assess the baseline of spontaneous seizure activity before drug injection (BoxA, $7.5 \mu \mathrm{g}$ per mouse or Lps-Rs, $5 \mu \mathrm{g}$ per mouse). EEG activity was then measured continuously after drug injection, and data were binned in 2-h periods. BoxA and Lps-Rs were injected into the same mice, $5 \mathrm{~d}$ apart; mice were also injected with PBS $24 \mathrm{~h}$ after the last drug administration. (b) Representative EEG tracings from mice with spontaneous seizures recorded during the pre-injection baseline, during the first $2 \mathrm{~h}$ after Lps-Rs or BoxA injection, and during the 4-h period before ending the experiment (post-injection baseline). Ictal activity is delimited by arrowheads. RHP and LHP, right and left hippocampus. (c) Quantification of BoxA and Lps-Rs effects on chronic spontaneous seizures. Error bars represent the means \pm s.e.m. $(n=8)$ of ictal activity in each mouse, expressed as percentage of the corresponding pre-injection baseline (raw data in Supplementary Fig. 2 ). ${ }^{* *} P<0.01$ versus baseline by repeated measures ANOVA followed by Dunnett's test.

reduced the frequency and duration of kainic acid-induced seizures (Fig. $4 \mathbf{b}, \mathbf{c}$ ); a dose of $0.8 \mu \mathrm{g}$ was ineffective.

We then tested two TLR4 antagonists, Rhodobacter sphaeroides LPS $(\text { Lps-Rs })^{37}$ and cyanobacterial LPS (Cyp) ${ }^{38}$. Lps-Rs had anticonvulsant effects already at $2 \mu \mathrm{g}$ per mouse (Fig. $4 \mathbf{a}-\mathbf{c}$ ). Both antagonists at $5 \mu \mathrm{g}$ per mouse substantially ameliorated all parameters of kainic acid-induced seizures (Fig. 4a-c).

BoxA and Lps-Rs were also highly effective in the bicuculline model; mice experienced substantially fewer seizures, spent less time in seizures and seizure onset was delayed (Fig. $4 \mathbf{d}-\mathbf{f}$ ).

Finally, we targeted the HMGB1-TLR4 axis in the model of chronic spontaneous seizures (Fig. 5). We recorded EEG activity in C57BL/6 mice with a stable baseline of spontaneous seizures a
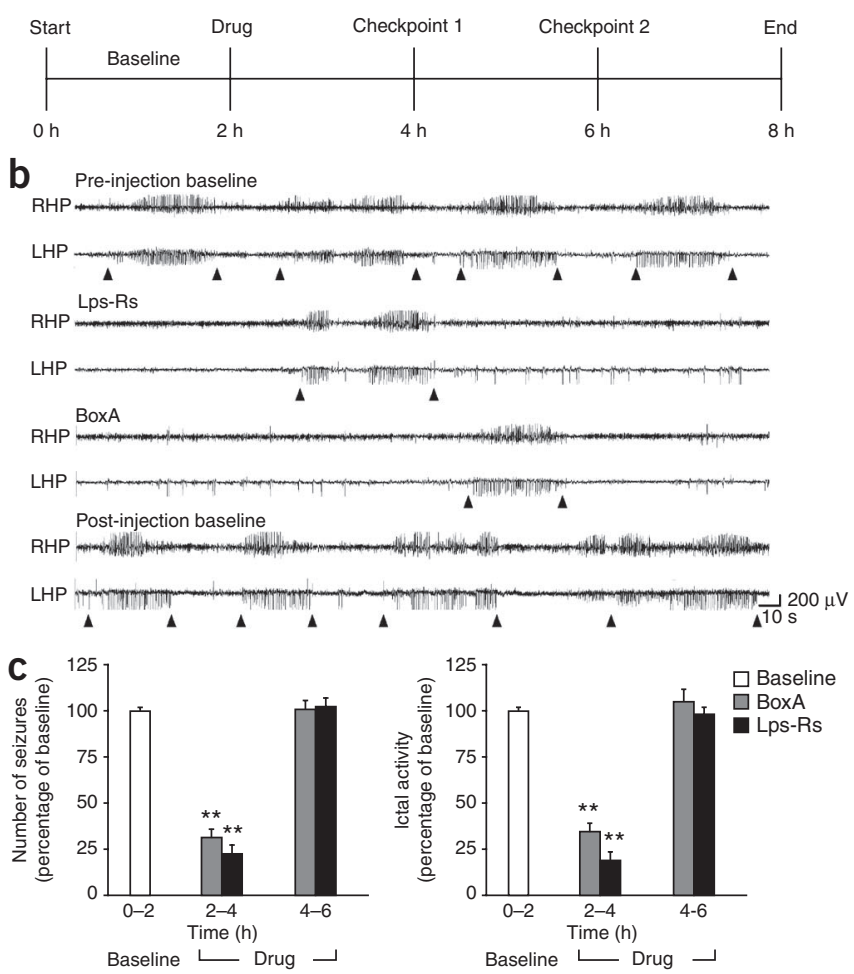

before and after injection of BoxA (7.5 $\mu$ g per mouse) or Lps-Rs (5 $\mu \mathrm{g}$ per mouse). Each antagonist lowered the number and frequency of spontaneous seizures up to $\sim 75 \%$ for about $2 \mathrm{~h}$ after administration (Fig. 5c).

\section{a}

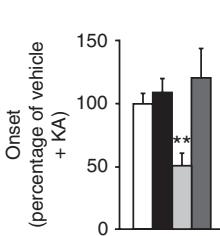

$\square$ Vehicle + KA

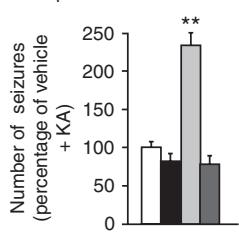

$$
\square
$$

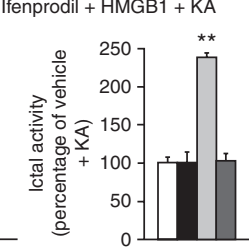

b

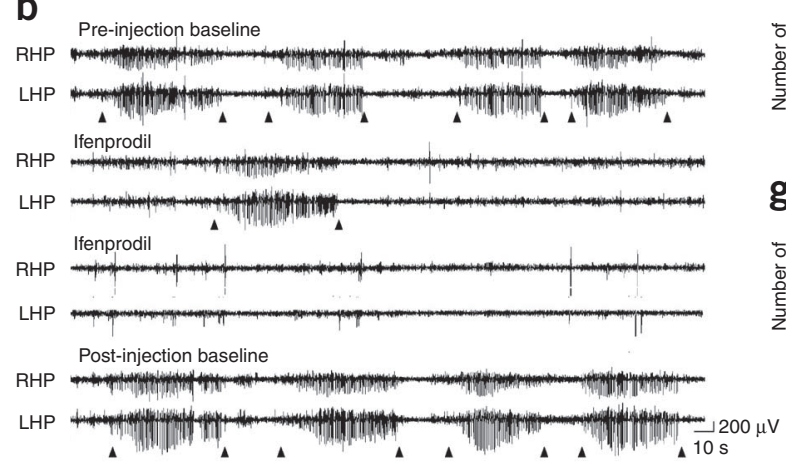

C

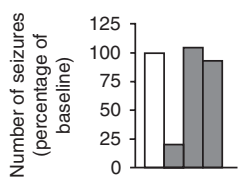

e.
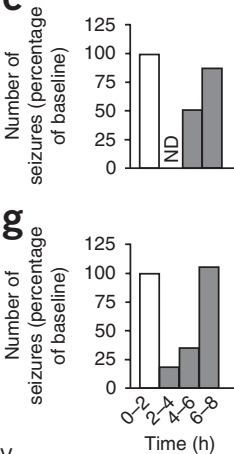

d
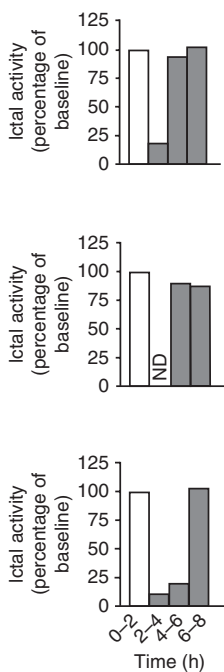

f

h
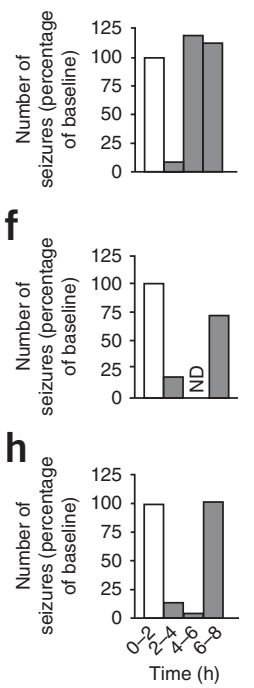

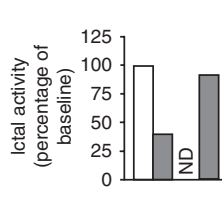

$\square$ Baseline
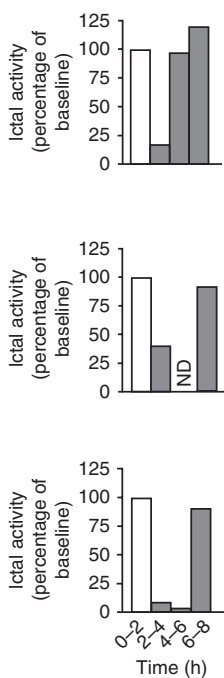

Figure 6 Effect of ifenprodil on acute and chronic seizures. (a) Acute seizure parameters (onset, number and duration) in C57BL/6 mice injected intrahippocampally with HMGB1 (9.5 $\mu$ g per mouse, $n=8$ per group) or vehicle ( $n=10$ per group) and intraperitoneally with ifenprodil ( $1 \mathrm{mg}$ per kg body weight) where indicated. HMGB1 was injected $15 \mathrm{~min}$, and ifenprodil $20 \mathrm{~min}$, before intrahippocampal kainic acid injection. Error bars represent means \pm s.e.m. normalized to the vehicle + KA group (onset $8.7 \pm 0.9 \mathrm{~min}$; number of seizures $10.0 \pm 1.0$; ictal activity $6.6 \pm 1.0$ min). ${ }^{* *} P<0.01$ versus vehicle + KA by two-way ANOVA followed by Tukey's test. (b) Representative EEG tracings recorded in mice with chronic spontaneous seizures during the pre-injection baseline, during the first $2 \mathrm{~h}$ period after ifenprodil i.p. injection (40 mg per $\mathrm{kg}$ body weight) and the following 4-h period (post-injection baseline). Ictal activity is delimited by arrowheads. (c-h) Ifenprodil effects on chronic spontaneous seizures. Bars represent seizure parameters in each of six chronic epileptic mice, expressed as percentage of the corresponding pre-injection baseline in the same mouse (raw data in Supplementary Fig. 2). ND, not detectable. 


\section{NR2B as a molecular target of HMGB1-TLR4 signaling}

IL-1 $\beta$, upon binding IL-1R1, exerts its proconvulsant effects by activating NR2B-containing NMDA receptors ${ }^{12,15,16}$. Accordingly, ifenprodil, a selective blocker of NR2B-containing NMDA receptors ${ }^{39}$, abrogates the proconvulsant activity of IL- $1 \beta^{16}$. As both TLR4 and IL-1R1 signaling depend on the MyD88 adapter protein, we investigated whether HMGB1 acts like IL-1 $\beta$. Ifenprodil injected together with kainic acid and HMGB1 eliminated the proconvulsant effect of HMGB1, returning all seizure parameters to the values observed in mice injected with kainic acid alone (Fig. 6a). As shown for IL-1 $\beta^{15,16}$, HMGB1 increased NR2B phosphorylation by 1.7-fold, as assessed by western blotting of hippocampal homogenates, comparable to the effect of kainic acid alone (not shown).

However, ifenprodil had no effect on seizures induced by kainic acid alone. We administered ifenprodil either intraperitoneally ( $40 \mathrm{mg}$ per $\mathrm{kg}$ body weight $\left.{ }^{40}\right)$, intracerebroventricularly $\left(80 \mu \mathrm{mol}\right.$ per mouse $\left.{ }^{41}\right)$ or intrahippocampally ( 50 pmol per mouse ${ }^{42}$ ) before injecting kainic acid, but none of these experimental regimens affected seizure parameters (data not shown). We thus hypothesized that ifenprodil might affect ictogenesis only in the context of an inflammatory milieu, as mimicked by exogenous application of HMGB1 and as typical of the hippocampus of chronically epileptic mice (Supplementary Fig. 2). Indeed, ifenprodil administered intraperitoneally ( $40 \mathrm{mg}$ per kg body weight) reduced by $60-100 \%$ the number and duration of spontaneous seizures in each of six chronically epileptic mice (Fig. 6b-h).

\section{DISCUSSION}

This study identifies the involvement of HMGB1 and TLR4 in the generation and recurrence of seizures and describes previously unrecognized inhibitors of ictal activity. We used two models of acute seizures (kainic acid-induced and bicuculline-induced) and one chronic model of spontaneous recurrent seizures. We also show that the histopathological patterns of HMGB1 and TLR4 expression in the mouse model of spontaneous recurrent seizures and in hippocampal specimens from subjects with TLE are very similar.

The experimental evidence for the involvement of the HMGB1TLR4 axis in seizures is based on the anticonvulsant activity of TLR4 inhibitors and BoxA, a competitor of endogenous HMGB1. Moreover, mutant mice with impaired TLR4 signaling do not respond to HMGB1 and are spontaneously resistant to kainic acid-induced seizures.

TLR4 activation by LPS, mimicking bacterial infection, decreases seizure threshold in adult and immature mice and rats ${ }^{19,20}$ and is associated with a chronic increase in hippocampal neuronal network excitability $^{20}$. In these studies, the effects of LPS developed over a time frame of several hours to weeks and were attributed to the transcriptional activation of inflammatory or neuronal plasticity genes $^{43}$ in response to PAMPs. A recent report showed that LPS may cause epileptiform activity in rats, with a rapid time of onset ${ }^{44}$. In our studies, TLR4-mediated effects in acute seizures occur in less than $10 \mathrm{~min}$ and are initiated by HMGB1, a DAMP released by damaged or highly stressed cells, which acts as the TLR4 ligand. Although seizure susceptibility can be increased by infective agents ${ }^{45}$, epileptic activity usually occurs in sterile conditions where PAMPs are not present. DAMPs, and HMGB1 in particular, are therefore likely to have a key role in seizure precipitation and recurrence. HMGB1 might also contribute to the known association between brain injury and epilepsy ${ }^{46}$.

The effects of HMGB1 and IL- $1 \beta$ seem very similar, especially because both are blocked by ifenprodil (as shown here and in and refs. 15,16), a selective antagonist of NR2B-containing NMDA receptors ${ }^{47}$. Whereas the HMGB1-TLR4 axis contributes to both acute and chronic seizures, ifenprodil has no effect on acute seizures but substantially reduces the frequency and duration of spontaneous seizures in chronic epileptic mice. We suggest that NR2B-containing NMDA receptors are not involved in the ictogenic activity of the HMGB1-TLR4 axis unless a preexisting or persistent inflammatory milieu is present, as occurs in chronic epileptic tissue ${ }^{6-10}$ or as mimicked by preapplication of HMGB1.

Although it is clear that HMGB1 and TLR4 participate in the chain of events leading to the precipitation and recurrence of seizures, as indicated by our genetic and pharmacological studies, the source of HMGB1 is less clear and seems to change over time. Given that HMGB1 is present in the nuclei of principal neurons before convulsant challenge, we hypothesize that HMGB1 is released by pyramidal and granule neurons after or concomitantly with the release of glutamate (Supplementary Fig. 7). Indeed, our in vitro data clearly show that neurons undergoing excitotoxic death release HMGB1, suggesting that CA3 pyramidal cells killed by kainic acid can be a source of HMGB1. However, blockade of the HMGB1-TLR4 axis retards seizure onset also in the bicuculline-induced nonlesional model of seizures, suggesting that neuronal overexcitation itself is sufficient to promote HMGB1 release and that cell death is not a prerequisite. In addition to the release of preformed HMGB1 at seizure onset, new synthesis of HMGB1 occurs in the hippocampus after seizure precipitation, as shown by immunohistochemistry and western blotting. Concomitantly with new synthesis of HMGB1, we observe HMGB1 in the cytoplasm of astrocytes and microglia, suggesting that glial cells actively secrete HMGB1, although this is difficult to show in vivo. TLR4 expression after seizure precipitation is also increased from basal conditions in normal brain tissue. Although TLR4 protein is not detectable by immunohistochemistry in control tissue, TLR 4 transcript is present ${ }^{18}$, indicating a readily available machinery for protein upregulation.

The cause of HMGB1 relocation and synthesis in glia, and of TLR4 expression during seizures, remains unknown. We could not induce HMGB1 synthesis or secretion by rat microglia and astrocytes after incubation with kainic acid, glutamate or inflammatory mediators such as tumor necrosis factor- $\alpha$, IL-1 $\beta$, ATP or a combination of all three (data not shown). Hypoxia has been shown in several scenarios to cause HMGB1 relocation, synthesis or both ${ }^{36,48,49}$; however, only a temporary mild decrease in tissue oxygenation occurs in the epileptic focus in the early phase of ictal activity ${ }^{50}$. Moreover, hypoxia has been shown to induce transcriptional downregulation of TLR4 in cell cultures ${ }^{51}$.

In any event, increased HMGB1 expression and secretion by glial cells during seizures is expected to support a second and sustained wave of extracellular HMGB1, distinct from the first wave from overexcited neurons at seizure onset. Both waves possibly enforce an autocrine and paracrine loop supporting neuronal hyperexcitability and sustain inflammation via nuclear factor- $\kappa \mathrm{B}-$ and/or activator protein-1-dependent transcription of inflammatory genes, including IL-1 $\beta$. TLR4 induction in neurons and astrocytes can further support the efficacy and duration of this autocrine or paracrine loop.

The presence of cytoplasmic HMGB1 in a high percentage of glial cells and high TLR4 expression in brain specimens from subjects with TLE, who are highly prone to seizure precipitation, suggests that these molecules might have key roles in human epilepsy. Widespectrum anti-inflammatory drugs, such as steroids, are effective in some types of epilepsy ${ }^{3}$. However, HMGB1 and TLR4 may be optimal targets for antiepileptic treatments; indeed, HMGB1 and TLR4 inhibitors are active in the mouse model of chronic seizures, which is resistant to a range of anticonvulsant drugs ${ }^{29}$. Pharmacological interventions targeting HMGB1 are already under development for several disorders ${ }^{52}$ and may prove effective for epilepsies that are currently drug resistant. 


\section{METHODS}

Methods and any associated references are available in the online version of the paper at http://www.nature.com/naturemedicine/.

Note: Supplementary information is available on the Nature Medicine website.

\section{ACKNOWLEDGMENTS}

This work was supported by a CARIPLO Foundation grant (A.V., M.E.B. and C.R.), by Parents Against Childhood Epilepsy (A.V.), Associazione Italiana Contro l'Epilessia (T.R.), EU FP6 project EPICURE (LSH-CT-2006-037315) (A.V.), EU FP7 project NeuroGlia (No. 202167) and National Epilepsy Fund (NEF 09-05) (E.A.) and a grant from Regione Lombardia to HMGBiotech. J.L. is a recipient of an EU fellowship in the International Graduate Programme in Molecular Medicine training program. We acknowledge F. De Ceglie for technical support in photograph preparation.

\section{AUTHOR CONTRIBUTIONS}

M. Maroso and S.B. conducted the pharmacological studies in the experimental models of seizures and performed the analysis of data; T.R. conducted the immunohistochemical analysis in the experimental models and analyzed the data; J.L. performed the western blot analyses and in vitro experiments; E.A. and A.M.I. conducted the immunohistochemical studies in human tissues and data analysis; C.R. and M. Molteni characterized and provided the TLR4 antagonists used in the pharmacological experiments; M.C. produced HMGB1 and BoxA;A.A.M. contributed experimental suggestions and participated in manuscript editing; M.E.B. together with A.V. supervised all phases of the project and wrote the manuscript.

\section{COMPETING FINANCIAL INTERESTS}

The authors declare competing financial interests: details accompany the full-text HTML version of the paper at http://www.nature.com/naturemedicine/.

\section{Published online at http://www.nature.com/naturemedicine/.}

Reprints and permissions information is available online at http://npg.nature.com/ reprintsandpermissions/.

1. Hauser, W.A., Annegers, J.F. \& Kurland, L.T. Incidence of epilepsy and unprovoked seizures in Rochester, Minnesota: 1935-1984. Epilepsia 34, 453-468 (1993).

2. Perucca, E., French, J. \& Bialer, M. Development of new antiepileptic drugs: challenges, incentives, and recent advances. Lancet Neurol. 6, 793-804 (2007).

3. Vezzani, A. \& Granata, T. Brain inflammation in epilepsy: experimental and clinical evidence. Epilepsia 46, 1724-1743 (2005)

4. Pitkänen, A. \& Sutula, T.P. Is epilepsy a progressive disorder? Prospects for new therapeutic approaches in temporal-lobe epilepsy. Lancet Neurol. 1, 173-181 (2002).

5. Bartfai, T. et al. Interleukin-1 system in CNS stress: seizures, fever and neurotrauma. Ann. NY Acad. Sci. 1113, 173-177 (2007).

6. Sheng, J.G., Boop, F.A., Mrak, R.E. \& Griffin, W.S. Increased neuronal $\beta$-amyloid precursor protein expression in human temporal lobe epilepsy: association with interleukin-1 $\alpha$ immunoreactivity. J. Neurochem. 63, 1872-1879 (1994).

7. Crespel, A. et al. Inflammatory reactions in human medial temporal lobe epilepsy with hippocampal sclerosis. Brain Res. 952, 159-169 (2002).

8. Ravizza, T. et al. Innate and adaptive immunity during epileptogenesis and spontaneous seizures: evidence from experimental models and human temporal lobe epilepsy. Neurobiol. Dis. 29, 142-160 (2008).

9. Ravizza, T. et al. The IL-1 $\beta$ system in epilepsy-associated malformations of cortical development. Neurobiol. Dis. 24, 128-143 (2006).

10. Boer, K. et al. Inflammatory processes in cortical tubers and subependymal giant cell tumors of tuberous sclerosis complex. Epilepsy Res. 78, 7-21 (2008).

11. Vezzani, A., Balosso, S. \& Ravizza, T. The role of cytokines in the pathophysiology of epilepsy. Brain Behav. Immun. 22, 797-803 (2008).

12. Vezzani, A. et al. Interleukin-1 $\beta$ immunoreactivity and microglia are enhanced in the rat hippocampus by focal kainate application: functional evidence for enhancement of electrographic seizures. J. Neurosci. 19, 5054-5065 (1999).

13. De Simoni, M.G. et al. Inflammatory cytokines and related genes are induced in the rat hippocampus by limbic status epilepticus. Eur. J. Neurosci. 12, 2623-2633 (2000).

14. Ravizza, T. et al. Interleukin converting enzyme inhibition impairs kindling epileptogenesis in rats by blocking astrocytic IL-1 $\beta$ production. Neurobiol. Dis. 31, 327-333 (2008)

15. Viviani, B. et al. Interleukin-1ß enhances NMDA receptor-mediated intracellular calcium increase through activation of the Src family of kinases. J. Neurosci. 23, 8692-8700 (2003).

16. Balosso, S. et al. A novel non-transcriptional pathway mediates the proconvulsive effects of interleukin-1 1 . Brain 131, 3256-3265 (2008).

17. Kawai, T. \& Akira, S. Signaling to NF-kB by Toll-like receptors. Trends Mol. Med. 13, 460-469 (2007).

18. Chakravarty, S. \& Herkenham, M. Toll-like receptor 4 on nonhematopoietic cells sustains CNS inflammation during endotoxemia, independent of systemic cytokines. J. Neurosci. 25, 1788-1796 (2005).
19. Sayyah, M., Javad-Pour, M. \& Ghazi-Khansari, M. The bacterial endotoxin lipopolysaccharide enhances seizure susceptibility in mice: involvement of proinflammatory factors: nitric oxide and prostaglandins. Neuroscience 122 1073-1080 (2003)

20. Galic, M.A. et al. Postnatal inflammation increases seizure susceptibility in adult rats. J. Neurosci. 28, 6904-6913 (2008).

21. Bianchi, M.E. \& Manfredi, A.A. Immunology. Dangers in and out. Science 323, 1683-1684 (2009)

22. Scaffidi, P., Misteli, T. \& Bianchi, M.E. Release of chromatin protein HMGB1 by necrotic cells triggers inflammation. Nature 418, 191-195 (2002).

23. Müller, S., Ronfani, L. \& Bianchi, M.E. Regulated expression and subcellular localization of HMGB1, a chromatin protein with a cytokine function. J. Intern. Med. 255, 332-343 (2004).

24. Bonaldi, T. et al. Monocytic cells hyperacetylate chromatin protein HMGB1 to redirect it towards secretion. EMBO J. 22, 5551-5560 (2003).

25. Park, J.S. et al. Involvement of Toll-like receptors 2 and 4 in cellular activation by high mobility group box 1 protein. J. Biol. Chem. 279, 7370-7377 (2004).

26. Apetoh, L. et al. Toll-like receptor 4-dependent contribution of the immune system to anticancer chemotherapy and radiotherapy. Nat. Med. 13, 1050-1059 (2007).

27. Ravizza, T. et al. Inactivation of caspase-1 in rodent brain: a novel anticonvulsive strategy. Epilepsia 47, 1160-1168 (2006).

28. Vezzani, A. et al. Powerful anticonvulsant action of IL-1 receptor antagonist on intracerebral injection and astrocytic overexpression in mice. Proc. Natl. Acad. Sci. USA 97, 11534-11539 (2000).

29. Riban, V. et al. Evolution of hippocampal epileptic activity during the development of hippocampal sclerosis in a mouse model of temporal lobe epilepsy. Neuroscience 112, 101-111 (2002).

30. Bouilleret, V. et al. Recurrent seizures and hippocampal sclerosis following intrahippocampal kainate injection in adult mice: electroencephalography, histopathology and synaptic reorganization similar to mesial temporal lobe epilepsy. Neuroscience 89, 717-729 (1999).

31. Poltorak, A. et al. Defective LPS signaling in $\mathrm{C} 3 \mathrm{H} / \mathrm{HeJ}$ and $\mathrm{C} 57 \mathrm{BL} / 10 \mathrm{ScCr}$ mice mutations in Tlr4 gene. Science 282, 2085-2088 (1998).

32. Trisciuoglio, L. \& Bianchi, M.E. Several nuclear events during apoptosis depend on caspase-3 activation but do not constitute a common pathway. PLoS One 4, e6234 (2009).

33. Yu, S.W. et al. Mediation of poly(ADP-ribose) polymerase-1-dependent cell death by apoptosis-inducing factor. Science 297, 259-263 (2002).

34. Yang, $\mathrm{H}$. et al. Reversing established sepsis with antagonists of endogenous highmobility group box 1. Proc. Natl. Acad. Sci. USA 101, 296-301 (2004).

35. Sitia, G., lannacone, M., Muller, S., Bianchi, M.E. \& Guidotti, L.G. Treatment with HMGB1 inhibitors diminishes CTL-induced liver disease in HBV transgenic mice. J. Leukoc. Biol. 81, 100-107 (2007).

36. Muhammad, S. et al. The HMGB1 receptor RAGE mediates ischemic brain damage J. Neurosci. 28, 12023-12031 (2008).

37. Kutuzova, G.D., Albrecht, R.M., Erickson, C.M. \& Qureshi, N. Diphosphoryl lipid A from Rhodobacter sphaeroides blocks the binding and internalization of lipopolysaccharide in RAW 264.7 cells. J. Immunol. 167, 482-489 (2001).

38. Macagno, A. et al. A cyanobacterial LPS antagonist prevents endotoxin shock and blocks sustained TLR4 stimulation required for cytokine expression. J. Exp. Med. 203, 1481-1492 (2006).

39. Chenard, B.L. \& Menniti, F.S. Antagonists selective for NMDA receptors containing the NR2B subunit. Curr. Pharm. Des. 5, 381-404 (1999).

40. Ebert, U., Wlaz, P. \& Loscher, W. Anticonvulsant effects by combined treatment with a glycineB receptor antagonist and a polyamine site antagonist in amygdalakindled rats. Eur. J. Pharmacol. 322, 179-184 (1997).

41. Yourick, D.L., Repasi, R.T., Rittase, W.B., Staten, L.D. \& Meyerhoff, J.L. Ifenprodil and arcaine alter amygdala-kindling development. Eur. J. Pharmacol. 371, 147-152 (1999).

42. De Sarro, G.B., Bagetta, G., Spagnolo, C. \& Nistico, G. Antagonists of N-methyl$\mathrm{D}$-aspartate receptors block seizures induced by putrescine in the deep prepiriform cortex. Neuropharmacology 32, 43-50 (1993).

43. Lee, M.S. \& Kim, Y.J. Signaling pathways downstream of pattern-recognition receptors and their cross talk. Annu. Rev. Biochem. 76, 447-480 (2007).

44. Rodgers, K.M. et al. The cortical innate immune response increases local neuronal excitability leading to seizures. Brain 132, 2478-2486 (2009).

45. Singh, G., Prabhakar, S. \& Modi, M. Central nervous system infections and epilepsy. Epilepsia 49 Suppl 6, 1 (2008).

46. Prince, D.A. et al. Epilepsy following cortical injury: cellular and molecular mechanisms as targets for potential prophylaxis. Epilepsia 50 Suppl 2, 30-40 (2009).

47. Yu, X.M., Askalan, R., Keil, G.J. II \& Salter, M.W. NMDA channel regulation by channel-associated protein tyrosine kinase Src. Science 275, 674-678 (1997).

48. Tsung, A. et al. HMGB1 release induced by liver ischemia involves Toll-like receptor 4 dependent reactive oxygen species production and calcium-mediated signaling. J. Exp. Med. 204, 2913-2923 (2007).

49. Andrassy, M. et al. High-mobility group box-1 in ischemia-reperfusion injury of the heart. Circulation 117, 3216-3226 (2008).

50. Zhao, M., Ma, H., Suh, M. \& Schwartz, T.H. Spatiotemporal dynamics of perfusion and oximetry during ictal discharges in the rat neocortex. J. Neurosci. 29, 2814-2823 (2009).

51. Ishida, I. et al. Hypoxia diminishes Toll-like receptor 4 expression through reactive oxygen species generated by mitochondria in endothelial cells. J. Immunol. 169, 2069-2075 (2002)

52. Yang, H., Wang, H., Czura, C.J. \& Tracey, K.J. HMGB1 as a cytokine and therapeutic target. J. Endotoxin Res. 8, 469-472 (2002). 


\section{ONLINE METHODS}

Experimental mice. We used male C57BL/6 mice, C3H/HeJ Tlr $4^{\mathrm{Lps}-\mathrm{d}}$ mutant mice and their corresponding wild-type $\mathrm{C} 3 \mathrm{H} / \mathrm{HeouJ}$ mice (60-d-old, $25 \mathrm{~g}$, Harlan) in conformance with institutional guidelines of the Mario Negri Institute for Pharmacological Research that are in compliance with Italian and international laws and policies. All experimental procedures were reviewed and approved by the Internal Animal Care and Use Committee of the Mario Negri Institute for Pharmacological Research.

Acute models of seizures. We surgically implanted mice with hippocampal depth electrodes and with a unilateral injection guide cannula positioned on top of the dura mater ${ }^{16}$. One week after surgery, we recorded EEGs in freely moving mice. Briefly, after recording a baseline EEG activity for $30 \mathrm{~min}$, we induced acute seizures by intrahippocampal application of kainic acid (7 ng, SigmaAldrich) or bicuculline methiodide (51 ng, Sigma-Aldrich). These doses induce recurrent EEG epileptiform activity in $100 \%$ of mice without mortality ${ }^{16,28}$. We dissolved the drugs in $0.5 \mu \mathrm{PBS}(\mathrm{pH} 7.4)$ and injected them unilaterally into the septal pole of the hippocampus $\left(0.5 \mu \mathrm{min}^{-1}\right)$ with a needle protruding $2.0 \mathrm{~mm}$ from the guide cannula and connected to a $10-\mu \mathrm{l}$ Hamilton microsyringe via PE20 tubing.

Kainic acid- and bicuculline-induced seizures elapsed within $120 \mathrm{~min}$ and $90 \mathrm{~min}$ from drug injection, respectively. We terminated EEG recordings after drug injection after we observed a 30-min EEG tracing similar to baseline.

Chronic model of spontaneous seizures. The chronic mouse model of spontaneous seizures has been extensively described ${ }^{29,30}$. Spontaneous seizures develop after nonconvulsive status epilepticus lasting for at least $3 \mathrm{~h}$, induced by unilateral intrahippocampal application (septal pole) of $200 \mathrm{ng}$ kainic acid in $50 \mathrm{nl}$ PBS. Spontaneous seizures reproducibly occur (Supplementary Fig. 2) 1 week, on average, after status epilepticus induction and recur for up to 8 months. The baseline of spontaneous seizures in each mouse is stable and reproducible, and seizures do not occur in clusters, as assessed by measuring the frequency and duration of EEG ictal events 2 months after induction of status epilepticus. This feature is a key prerequisite for reliable pharmacological studies. Spontaneous seizures in this model are refractory to various antiepileptic drugs, thus providing a valuable model for drug-resistant seizures ${ }^{29}$. Histopathological changes in the hippocampus are similar to those described in human mesial TLE including neuronal loss, granule cell dispersion, sprouting and inflammation $8,29,30,53,54$ (Supplementary Fig. 2).

For induction of status epilepticus, mice underwent stereotaxic surgery to place EEG recording depth electrodes, and we positioned a unilateral injection guide cannula on top of the dura mater for drug injections ${ }^{16}$. One week after surgery, we unilaterally injected kainic acid (200 $\mathrm{ng}$ in $50 \mathrm{nl}$ PBS) into the left septal hippocampus at the following coordinates from bregma: nose bar,
$0 \mathrm{~mm}$; anteroposterior, $-1.8 \mathrm{~mm}$, lateral, $1.7 \mathrm{~mm}$; and $1.9 \mathrm{~mm}$ below dura ${ }^{55}$. We injected kainic acid over 1 min (CMA/100 pump) with a 30-gauge injection needle connected to a $0.5-\mu 1$ Hamilton microsyringe via PE20 tubing. At the end of the injection, we left the needle in situ for an additional $2 \mathrm{~min}$ to limit backflow along the injection track. After kainic acid injection, we recorded EEG activity in the mice to assess status epilepticus for at least $3 \mathrm{~h}$, and then we returned them to their home cages until spontaneous seizure evaluation (2 months after status epilepticus).

To evaluate spontaneous seizures, we EEG-monitored mice every day from 9:30 to 17:30, for 5 consecutive days, to establish the baseline of spontaneous seizures. We quantified EEG seizures every $2 \mathrm{~h}$ on each day of recording. These recordings provided the daily average number of ictal events and their duration occurring in each mouse within the time-frame interval of our pharmacological experiments. We subsequently used mice that developed a stable baseline of chronic spontaneous seizures $(80 \%)$ for pharmacological experiments (Supplementary Fig. 2f-m). The next day, we recorded EEGs for $2 \mathrm{~h}$ and then injected the mice into the left septal hippocampus with BoxA or Lps-Rs or intraperitoneally with ifenprodil at the doses indicated in the main text or the figure legends (Fig. 5 and Fig. 6b) and monitored EEG activity for the subsequent $6 \mathrm{~h}$. We binned the data in 2-h periods. The same mice received the three drugs (Lps-Rs, BoxA and ifenprodil), each separated by a $5-\mathrm{d}$ washout period that allowed the full recovery of baseline parameter of spontaneous seizures. Finally, we injected each mouse intrahippocampally with $1 \mu \mathrm{l}$ PBS and recorded EEG activity for $6 \mathrm{~h}$ to verify activity after sham drug injection. Epileptic mice received a corresponding volume of vehicle intraperitoneally $24 \mathrm{~h}$ after ifenprodil injection. We did not observe any effects of vehicle injection on spontaneous seizure activity in the mice.

In each mouse, we evaluated the correct position of the electrodes and injection needle in the sections used for immunohistochemical analysis of brain tissue.

Additional methodology. EEG analysis of seizures, drug administration schedule, immunohistochemical procedures, clinical information, cell quantification in experimental and human specimens, western blot analysis, in vitro experiments of HMGB1 release and statistical analysis of data is described in the Supplementary Methods.

53. Kralic, J.E., Ledergerber, D.A. \& Fritschy, J.M. Disruption of the neurogenic potentia of the dentate gyrus in a mouse model of temporal lobe epilepsy with focal seizures. Eur. J. Neurosci. 22, 1916-1927 (2005).

54. Suzuki, F. et al. Glutamate receptor antagonists and benzodiazepine inhibit the progression of granule cell dispersion in a mouse model of mesial temporal lobe epilepsy. Epilepsia 46, 193-202 (2005).

55. Paxinos, G. \& Franklin, K.B.J. The Mouse Brain in Stereotaxic Coordinates. (Academic Press, San Diego, 1997). 